\title{
La revitalisation urbaine au sein des villes petites et moyennes. Quelques projets dans la région du Lac-Saint-Jean.
}

\author{
Martin Simard ${ }^{1}$ et Simon Ouellet ${ }^{2}$ \\ Université du Québec à Chicoutimi
}

\section{Introduction}

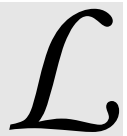
es villes petites et moyennes ont été identifiées comme étant des éléments essentiels de l'organisation socio-économique du Québec $^{3}$. Ce rôle semble particulièrement important en ce qui concerne les villes de ce type situées hors des aires de rayonnement de Québec et de Montréal. En effet, en plus de permettre l'occupation du territoire ainsi que l'exploitation et la transformation des ressources, les villes petites et moyennes assurent la prestation de services gouvernementaux et commerciaux à la population sur l'ensemble des territoires. D'une certaine façon, celles-ci participent à la mise en œuvre du modèle québécois en favorisant l'équité dans la diversité des services et la qualité de vie offerte aux citoyens.

La fonction socio-économique des villes de taille modeste passe notamment par le maintien d'un centreville actif et dynamique. À cet égard, les centresvilles des villes petites et moyennes ont été fortement affectés par la concurrence des centres commerciaux périurbains à partir des années 1970. En effet, la faible taille de ces centres-villes n'a pas permis de faire contrepoids aux centres d'achats qui agglomèrent des dizaines de commerces à l'intérieur de milieux climatisés pourvus de vastes stationnements.

Depuis une dizaine d'années, le phénomène des magasins à grandes surfaces de type Wal-Mart a accentué le dépérissement du centre dans plusieurs villes petites et moyennes. Toutefois, cette situation a eu le mérite de soulever des débats sur l'avenir du centreville au sein de plusieurs communautés locales.
Dans ce contexte, on remarque un regain d'intérêt envers les espaces urbains traditionnels chez les populations et les agents économiques. Ce phénomène est canalisé par les mouvements du Smarth Growth et du New Ubanism au Canada et aux États-Unis. Les centres-villes sont décrits par plusieurs auteurs comme étant des milieux animés à caractère humain qui se distinguent de la froideur des centres d'achats. Leur destinée ne serait pas de remplacer les centres commerciaux par des aménagements favorables aux automobiles, mais plutôt de chercher à se distinguer en jouant la carte de l'atmosphère urbaine et des produits spécialisés ${ }^{4}$. Ces éléments sont à la base de ce qu'on peut appeler la revitalisation urbaine de troisième génération. Celle-ci se différencie des approches des décennies précédentes fondées essentiellement sur l'accessibilité automobile et le stationnement (première génération) ainsi que sur l'attraction des commerces de type major afin de faire compétition aux centres commerciaux ( $2^{\mathrm{e}}$ génération) ${ }^{5}$.

Cet article vise à faire le point sur les stratégies de revitalisation urbaine utilisées par les instances municipales au sein de quatre villes de la région du LacSaint-Jean au Québec, soit Alma, Dolbeau-Mistassini, Saint-Félicien et Roberval. Il s'agit de comparer le contexte et les stratégies utilisées à l'intérieur de ces villes de taille similaire situées à relative proximité l'une de l'autre. Nous pourrons également observer dans quelle mesure ces interventions s'inspirent des tendances actuelles en matière de revitalisation.

Pour ce faire, nous avons rencontré différents intervenants locaux et analysé divers documents d'urbanis- 
me. Des coupures de presse du journal Le Quotidien ont aussi été consultées au cours de 2004 et de 2005.

\section{La revitalisation urbaine : quelques réflexions théoriques}

Qu'on l'appelle rénovation urbaine, réhabilitation urbaine ou renouveau urbain, la revitalisation demeure une pratique urbanistique à l'avant-scène depuis près d'un demi-siècle. L'expression revitalisation urbaine intégrée a récemment été proposée pour mettre en relief une approche nouvelle. La revitalisation urbaine intégrée désigne les plans d'action sur les milieux urbains en dévitalisation qui se veulent multisectoriels, partenariaux et opérant à plusieurs échelles géographiques ${ }^{6}$. Cette pratique s'insère dans le paradigme du développement local en misant sur les processus participatifs et les petits projets. Celle-ci tente d'éviter les pièges de la revitalisation urbaine traditionnelle.

Les démarches réalisées au nom de la revitalisation urbaine ont souvent causé plus de tort que de bien, malgré les intentions louables des maîtres d'œuvre. En effet, diverses actions comme la démolition d'îlots résidentiels ainsi que la reconfiguration du réseau routier ont modifié considérablement la physionomie des centres-villes touchés. Le principal effet néfaste des pratiques traditionnelles de revitalisation a sans nul doute été les déplacements de populations par l'intermédiaire des démolitions ou du phénomène d'embourgeoisement ${ }^{7}$. La revitalisation urbaine traditionnelle se basait sur un diagnostic sévère à l'endroit des espaces urbains anciens : il fallait nettoyer ou assainir une portion malade de la ville, quitte à amputer.

Les pratiques actuelles de revitalisation se réclament d'une représentation collective plus positive à l'égard des centres-villes et des quartiers anciens. À partir d'un cadre d'analyse valorisant l'urbanité, les autorités publiques opèrent généralement dans une dynamique dialectique avec le marché en tentant de rendre le territoire local apte à recevoir de nouveaux investissements privés. On aspire à créer un phénomène d'attraction en développant des aménités particulières ou à l'aide de subventions associées à une localisation précise. Cette double technique fut utilisée dans les cas de la Cité du multimédia à Montréal et du quartier Saint-Roch à Québec.
Malgré l'évolution des méthodes, la relance des milieux urbains dévitalisés par l'intermédiaire de programmes provinciaux ou fédéraux coûte cher en fonds publics. Ces améliorations et investissements sont mis en place dans un court laps de temps afin de promouvoir l'idée de changement et de façonner une nouvelle image des lieux. Néanmoins, cet effet tend à s'estomper dans le temps. Les études et les interventions sont généralement à recommencer après cinq ou dix ans. De plus, les stratégies se limitent le plus fréquemment aux interventions physiques sur le domaine public (rues, trottoirs, parcs et bâtiments communautaires, etc.). Regardons si les projets de revitalisation urbaine dans la région du Lac-Saint-Jean paraissent appelés à surmonter ces écueils.

\section{Les projets de revitalisation dans la région du Lac-Saint-Jean}

La région du Lac-Saint-Jean est située au centre nord du Québec méridional à l'intérieur de la région administrative du Saguenay-Lac-Saint-Jean. L'écoumène en forme de beigne s'organise en fonction de la présence du célèbre lac qui est en fait un réservoir depuis 1927. L'espace humanisé se retrouve clairement délimité par les hautes terres du Bouclier canadien. Les quatre villes étudiées, soit Alma, Dolbeau-Mistassini, Saint-Félicien et Roberval, sont les principaux noyaux de peuplement, ceux-ci regroupant près de $60 \%$ de la population régionale qui s'établissait à 111500 habitants en 2001. Ces milieux ont tous entrepris des démarches de revitalisation urbaine au cours des dernières années.

\section{La revitalisation du centre-ville à Alma}

La ville d'Alma constitue le chef-lieu de la MRC de Lac-Saint-Jean-Est et de l'ensemble de la région avec une population de 30126 habitants en 2001, équivalant à $58 \%$ des résidents de la MRC. Celle-ci affiche une économie assez dynamique marquée par la construction récente d'une aluminerie d'Alcan. Sur le plan géographique, cette municipalité occupe l'île d'Alma ainsi que les rives nord et sud de la rivière Saguenay, laquelle se divise ici en deux segments: la Grande décharge et la Petite décharge.

Le domaine commercial est particulièrement étendu à Alma. On retrouve ce type d'activités principalement au centre-ville et le long des boulevards Du Pont Sud 
et Du Pont Nord (figure 1). Le centre-ville se localise près de la Petite décharge, notamment autour des rues commerciales Sacré-Cœur et Collard. Autrement, on remarque plusieurs magasins à grande surface sur les artères périphériques, par exemple Rona, Canadian Tire, Wal-Mart, Gagnon \& frères ou Consomat, ainsi que trois centres d'achats, soit les Galeries Lac-SaintJean, la Place Saint-Luc et le Carrefour Alma.

\section{Figure 1 - Localisation du centre-ville d'Alma}

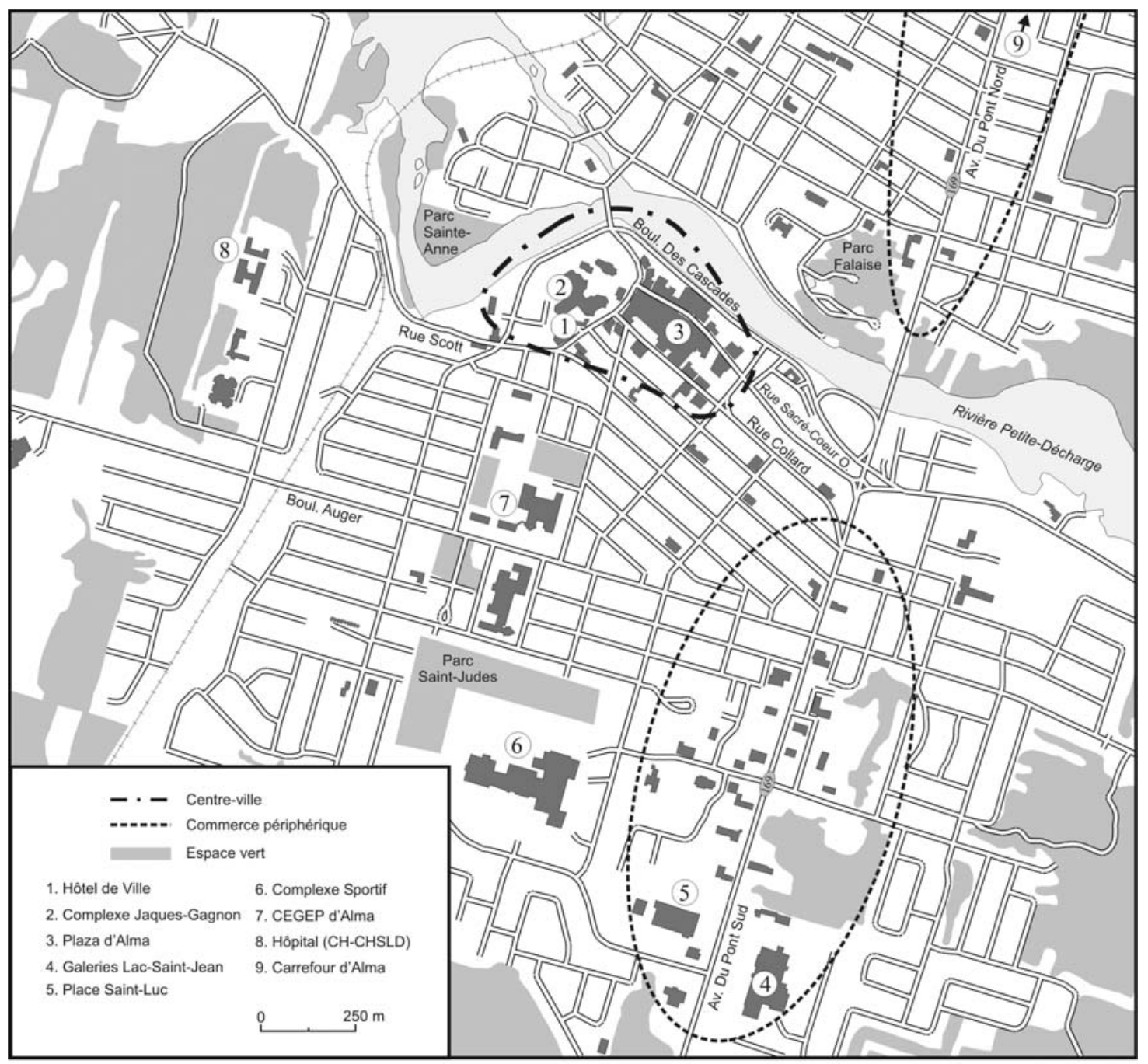

Le centre-ville d'Alma réunit plus de 300 commerces et services professionnels dont plus de la moitié est reliée au mail commercial de la Plaza d'Alma ${ }^{8}$. Plusieurs démarches de revitalisation ont été entreprises dans le passé : la réalisation du mail commercial de la Plaza d'Alma, au début des années 1970, et l'érection du complexe hôtelier Jacques-Gagnon, à la fin de la même décennie. Malgré cela, on comptait environ 80000 pieds carrés de locaux vacants au centre-ville à la fin de 2004. La société de développement des artères commerciales (SDC) d'Alma est l'organisme qui voit à promouvoir le commerce au centre-ville. La
SDC d'Alma représente 180 places d'affaires, totalisant environ 1200 employés.

D’après le schéma d'aménagement et de développement de la MRC de Lac-Saint-Jean-Est, la ville d'Alma est reconnue comme étant le pôle principal de commerces et de services. Plusieurs orientations du schéma sont associées à la revitalisation du centre-ville d'Alma, notamment l'orientation suivante : " Rendre le centre-ville et le secteur central de la municipalité plus attrayant en intervenant notamment au moyen de mesures réglementaires portant sur l'affi- 
chage, le revêtement extérieur, l'architecture et les aménagements paysagers $»^{9}$.

De son côté, la municipalité d'Alma a mis en place un plan de revitalisation du centre-ville visant à maintenir des activités commerciales diversifiées et attrayantes dans ce secteur. Selon nos sources, Alma a investi 2,7 millions de dollars entre 1996 et 2000 pour dynamiser ce territoire, améliorer les espaces publics, encourager la rénovation des bâtiments et favoriser l'occupation des locaux (tableau 1). Depuis l'adoption du plan d'urbanisme et du plan stratégique des interventions au centre-ville réalisés en 1996, la Ville d'Alma s'est principalement concentrée sur le projet de la nouvelle usine d'Alcan. De nombreux projets liés à la revitalisation du centre-ville ont été reportés, mais le nouveau plan d'urbanisme qui devrait être adopté en 2005 permettra de relancer ceux-ci.

\section{Tableau 1 - Liste des réalisations récentes et des projets au centre-ville d'Alma}

\begin{tabular}{|c|c|}
\hline Étape & Projet \\
\hline Réalisé & $\begin{array}{l}\text { Pont pour l'accès des motoneiges au } \\
\text { centre-ville }\end{array}$ \\
\hline Réalisé & Passerelle cyclable \\
\hline Réalisé & $\begin{array}{l}\text { Rétention des commerces et services au } \\
\text { centre-ville - exemple du CLSC }\end{array}$ \\
\hline Réalisé & $\begin{array}{l}\text { Événements : Drag de rue, Sacré Blues et } \\
\text { Septembre à vélo }\end{array}$ \\
\hline Réalisé & $\begin{array}{l}\text { Budget accordé à la SDC par la Ville pour } \\
\text { les activités culturelles }\end{array}$ \\
\hline Réalisé & $\begin{array}{l}\text { Mise en place d'un marché public et } \\
\text { amélioration de la signalisation }\end{array}$ \\
\hline Réalisé & Étude sur la mixité commerciale à Alma \\
\hline Projet & $\begin{array}{l}\text { Réaménagement des berges de la rivière } \\
\text { Petite-décharge }\end{array}$ \\
\hline
\end{tabular}

\section{La revitalisation des centres-villes à Dolbeau-Mistassini}

Dolbeau-Mistassini est le centre urbain le plus important au sein de la MRC Maria-Chapdelaine avec une population de 14879 habitants en 2001 (55\% de la MRC). Cette ville fusionnée en 1997 forme le deuxième pôle commercial en importance dans la région du Lac-Saint-Jean après Alma. Dolbeau-Mistassini a hérité d'un tissu urbain polycentrique. En effet, le centre-ville de Dolbeau-Mistassini est partagé en deux secteurs (figure 2). Le secteur de Dolbeau est organisé autour du boulevard Wallberg, entre la $3^{\mathrm{e}}$ et la $8^{\mathrm{e}}$ Avenue, dans un axe nord-sud, et entre les rues des Pins et des Érables, dans un axe est-ouest. Le centre-ville du secteur de Mistassini est situé sur le boulevard Saint-Michel entre le boulevard Panoramique et la rue de l'Église ainsi que sur la rue de l’Église, entre Saint-Michel et De Quen.

Plusieurs interventions ont ponctué le développement du centre-ville du secteur de Dolbeau au cours des deux dernières décennies, la plus connue étant la construction du controversé mail couvert sur le boulevard Wallberg, à la fin des années 1980. En plus de ce mail urbain, nous retrouvons au centre-ville de Dolbeau un supermarché Métro ainsi qu'une quincaillerie Pro Rénovation, sans compter le centre commercial Galeries des Érables qui est aussi situé dans la zone centre de ce secteur et qui contient un Canadian Tire, un Provigo et plusieurs autres commerces.

Du côté du secteur de Mistassini, les commerces sont également regroupés au centre-ville, le Mail centreville, le supermarché IGA, Gagnon \& frères et le nouveau Provigo étant les plus imposants. En périphérie du centre-ville du secteur de Mistassini, il y a une petite concentration commerciale sur la rue De Quen (route 169).

Le schéma d'aménagement et de développement de la MRC Maria-Chapdeleine identifie comme préoccupations majeures l'expansion du milieu urbain et la rentabilisation des services municipaux. Ces orientations militent en faveur de la réhabilitation des zones urbaines centrales. Pour sa part, la Ville de DolbeauMistassini a adopté en 2003 un plan stratégique pour le développement de la municipalité pour les années 2003, 2004 et 2005. La municipalité voudrait favoriser les activités commerciales au centre-ville du secteur de Dolbeau et promouvoir la vie culturelle et sociale dans le centre-ville du secteur de Mistassini.

La Ville de Dolbeau-Mistassini a également adopté par règlement un programme de revitalisation à l'égard des secteurs particuliers, tel le centre-ville (tableau 2). Cette municipalité veut consolider les liens entre les deux centres-villes en favorisant l'implantation de commerces et d'attractions touristiques le long du boulevard des Pères (route 169), notamment par la poursuite de l'aménagement du « Parc de la 
Pointe des Pères » dont le plan directeur a été réalisé en 2004. Par ailleurs, l'administration municipale dolmissoise veut accroître le rayonnement commercial du secteur de Dolbeau par l'ajout de magasins à grande surface à relative proximité du centre-ville, soit sur la $8^{\mathrm{e}}$ Avenue, par le développement de stationnements autour des Promenades du boulevard Wallberg.

L'administration municipale a initié la conception d'un programme incitatif pour la rénovation du parc immobilier commercial localisé dans les zones centres, dans l'optique de susciter un mouvement de restauration des façades de bâtiments. Ce programme prendra la forme d'une subvention par l'octroi d'un crédit de taxes foncières applicable aux travaux de construction. D'autre part, la rénovation des édifices résidentiels sera soutenue par l'adoption prochaine d'un plan d'implantation et d'intégration architecturale (PIIA) dans le quartier entourant la papetière, communément appelé le quartier des Anglais.
Tableau 2 - Liste des réalisations récentes et des projets aux centres-villes de Dolbeau-Mistassini

\begin{tabular}{cl} 
Étape & \multicolumn{1}{c}{ Projet } \\
\hline Réalisé & $\begin{array}{l}\text { Programme d’incitation à la rénovation par } \\
\text { crédits de taxe }\end{array}$ \\
\hline Réalisé & $\begin{array}{l}\text { Accès au centre-ville pour les } \\
\text { motoneigistes }\end{array}$ \\
\hline Réalisé & $\begin{array}{l}\text { Construction du manoir Cinq saisons } \\
\text { (personnes âgées) }\end{array}$ \\
\hline Réalisé & $\begin{array}{l}\text { Étude sur la circulation au centre-ville du } \\
\text { secteur de Dolbeau }\end{array}$ \\
\hline Projet & $\begin{array}{l}\text { Construction de canalisations de gaz } \\
\text { naturel }\end{array}$ \\
\hline Projet & Signalisation touristique \\
\hline Projet & $\begin{array}{l}\text { Aménagement du Parc de la pointe des } \\
\text { pères }\end{array}$ \\
\hline Projet & Construction d'une salle de spectacle \\
\hline &
\end{tabular}

Figure 2 - Localisation des centres-villes de Dolbeau-Mistassini

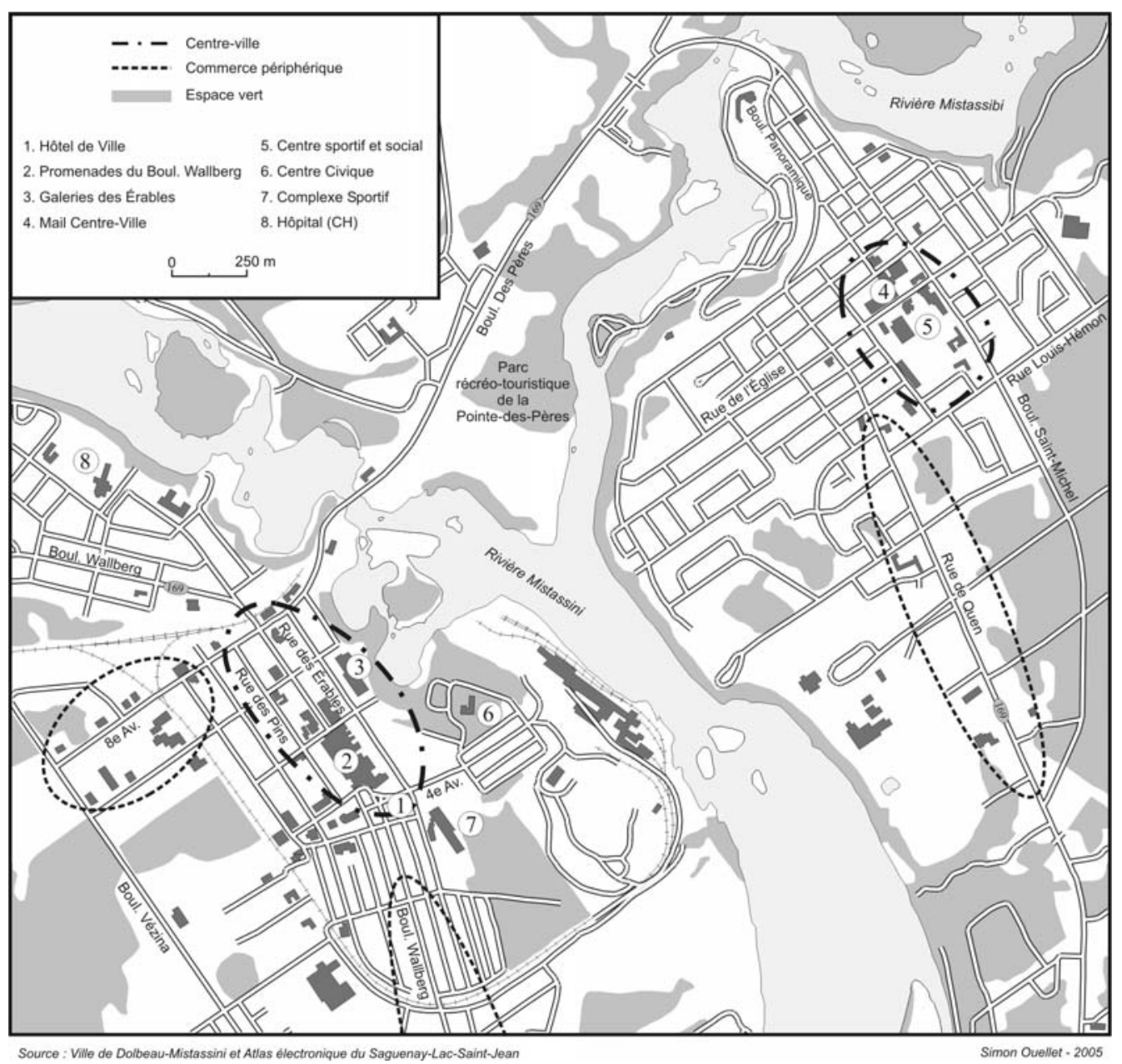




\section{La revitalisation du centre-ville à Saint-Félicien}

La ville de Saint-Félicien fait partie de la MRC du Domaine-du-Roy. Cette MRC comporte deux agglomérations ayant plus de 10000 habitants, soit Roberval et Saint-Félicien. Plus précisément, Saint-Félicien comptait 10622 habitants en 2001, soit $33 \%$ de la population de la MRC. À Saint-Félicien, le centre- ville est très bien défini par la signalisation. Celui-ci comprend la partie de la ville située entre le chemin de fer et la rivière Ashuapmushuan, de la rue SainteAnne au boulevard Saint-Félicien (figure 3). Ce territoire correspond au périmètre urbain de la ville de Saint-Félicien dans les années 1950, c'est-à-dire avant que le développement urbain ne déborde de l'autre côté de la voie ferrée du Canadien National.

Figure 3 - Localisation du centre-ville de Saint-Félicien

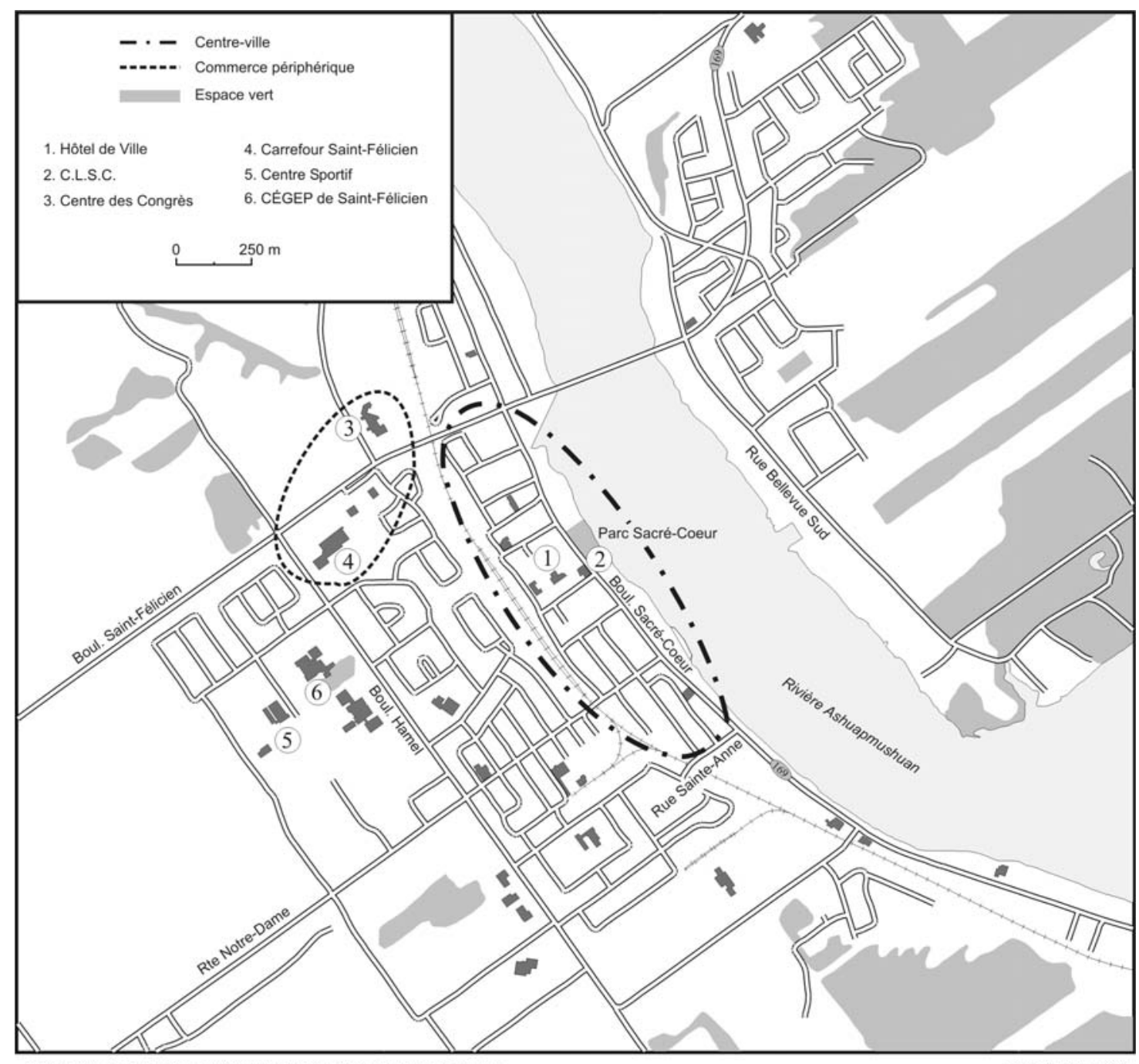

Source : Ville de Saint-Félicien et Atlas êlectronique du Saguenay-Lac-Saint-Jean

Simon Ouellet - 2005

Le centre-ville de Saint-Félicien renferme environ $40 \%$ des commerces localisés sur le territoire de la municipalité ${ }^{10}$. C'est la société de développement commercial (SDC) de Saint-Félicien qui supervise la promotion et le développement commercial au centreville. Il y a peu de commerces à grande surface à l'intérieur des limites du centre-ville, à l'exception du supermarché Provigo. Les magasins à grande surface et le centre d'achat Carrefour Saint-Félicien se retrouvent en périphérie du tissu urbain, aux abords du boulevard Saint-Félicien, à proximité du cégep du même nom et de la route d'accès au Zoo sauvage de Saint-Félicien.

Saint-Félicien est une municipalité active sur le plan de la revitalisation urbaine (tableau 3). Elle dispose 
d'un plan stratégique de revitalisation du centre-ville dont l'application est coordonnée par un chargé de projet du service d'urbanisme. La mise en œuvre de l'ensemble du programme d'intervention équivaudra à des investissements de quelques millions entre 2003 et 2007.

\section{Tableau 3 - Liste des réalisations récentes et des projets au centre-ville de Saint-Félicien ${ }^{11}$}

\begin{aligned} & \multicolumn{1}{c}{ Étape } \multicolumn{1}{c}{ Projet } \\ & \hline \multicolumn{1}{c}{ Réalisé } Élaboration d'une stratégie commerciale \\ & \hline Réalisé $\begin{array}{l}\text { Adoption d'un plan d'implantation et } \\ \text { d'intégration architecturale }\end{array} \\ &$\hline Réalisé $\begin{array}{l}\text { Élaboration du Plan directeur de } \\ \text { signalisation }\end{array} \\ &$\hline Réalisé $\begin{array}{l}\text { Mise en fonction d'instruments } \\ \text { financiers et fiscaux }\end{array} \\ &$\hline Réalisé $\begin{array}{l}\text { Réalisation de la place de l'hôtel de } \\ \text { ville }\end{array} \\ &$\hline Réalisé Rénovation de la maison de la Culture \\ & \hline En cours Rénovation du parc Sacré-Cœur \\ & \hline Projet Voie de contournement du centre-ville \\ & \hline Projet $\begin{array}{l}\text { Fontaine multimédia au parc Sacré- } \\ \text { Cœur }\end{array} \\ &$\hline Projet $\begin{array}{l}\text { Promenade aux abords de la rivière } \\ \text { Ashuapmushuan }\end{array} \\ &$\hline\end{aligned}

Avec l'aide de la Société canadienne d'hypothèques et de logement (SCHL) et de la Société d'habitation du Québec (SHQ), la Ville de Saint-Félicien a mis en place plusieurs programmes qui touchent principalement le secteur résidentiel (Logement Abordable Québec, Accès Logis Québec, crédits de taxes, embellissement des façades et subvention Rénovation Québec).

Avant de mettre en place la stratégie, la Ville de Saint-Félicien a créé un comité de revitalisation doté d'un budget de fonctionnement. De plus, l'administration municipale s'est associée à la Fondation Rues Principales pour s'inspirer des différentes démarches de revitalisation dans d'autres villes du Québec et du reste du Canada. Pour connaître les besoins de la population et des visiteurs, la municipalité a mené une campagne d'information, des inventaires et divers sondages. L'ensemble de la démarche semble porter fruit sur le plan commercial. En effet, depuis la mise en marche du plan d'action, près de vingt locaux vacants ont été comblés et un mouvement de retour vers le centre est observé. Par exemple, le magasin Radio Shack a quitté le centre d'achat pour s'installer au centre-ville. Finalement, Saint-Félicien veut augmenter le potentiel d'attraction de son centre-ville envers les touristes.

\section{La revitalisation du centre-ville à Roberval}

La ville de Roberval fait également partie de la MRC du Domaine-du-Roy. Il s'agit du premier pôle de la MRC avec une population de 10906 habitants en 2001, soit 33 \% de la population mercéenne. Établissement majeur au début du $\mathrm{XIX}^{\mathrm{e}}$ siècle, Roberval éprouve de la difficulté à maintenir sa position à l'intérieur du système urbain régional. La municipalité délimite son centre-ville par les voies ferrées et le lac Saint-Jean, dans un axe est-ouest, et par la rivière Ouiatchouaniche et l'hôpital de Roberval, dans un axe nord-sud (figure 4).

La répartition spatiale des commerces de Roberval démontre l'attraction de la périphérie par opposition au centre-ville. Le centre-ville de Roberval renferme seulement $28,6 \%$ des commerces situés sur le territoire de la municipalité ${ }^{12}$. En effet, nous observons 113 commerces au centre-ville comparativement à 284 en périphérie. Plusieurs magasins à grande surface tels que les supermarchés Maxi et IGA de même que la pharmacie Jean Coutu et la quincaillerie Rona, ainsi que le centre d'achats Le Carrefour Jeannois, se localisent aux abords des boulevards Marcotte et Saint-Dominique.

Entre 1975 et 1991, la MRC du Domaine-du-Roy a vu accroître le nombre de commerces ou places d'affaires de 56,2 \% pour atteindre un total de 917 . Cette hausse a été particulièrement importante dans les municipalités de Roberval et Saint-Félicien, soit 71,5 \%. Toutefois, la MRC du Domaine-du-Roy est d'avis que le déploiement des centres commerciaux en périphérie des noyaux urbains influence négativement le développement urbain sur son territoire, en particulier en ce qui concerne l'état de santé des centres-villes.

Le centre-ville de Roberval n'attire plus autant les commerces depuis que la route régionale (169) contourne le centre-ville par le biais du boulevard Marcotte. Les commerçants recherchent une plus grande visibilité en s'établissant en périphérie, en bordure de 
la route 169 et près du centre d'achats. Par contre, les intervenants municipaux observent une tendance contraire en ce qui a trait à certains commerces spécialisés et aux services professionnels, lesquels sont attirés par le centre ancien. Cela fait en sorte que le nombre de locaux vacants est sensiblement le même au centre-ville et en périphérie, soit une trentaine à l'intérieur de chacune de ces deux zones.

Figure 4 - Localisation du centre-ville de Roberval

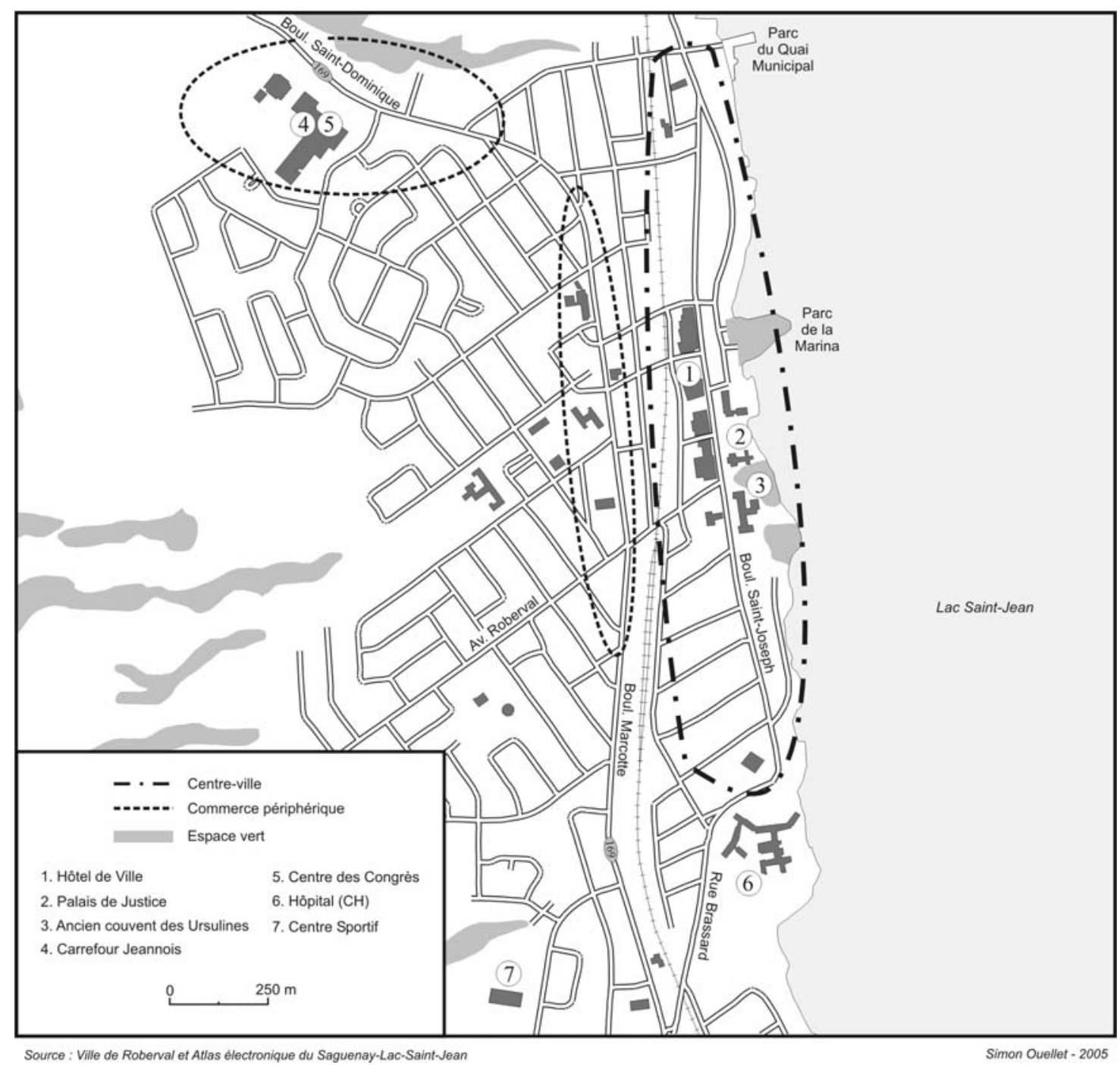

Il n'existe pas de Société d'initiative de développement des artères commerciales (SIDAC) ou de Société de développement commercial (SDC) sur la rue Saint-Joseph. Ce sont le Centre local de développement (CLD) et la Chambre de commerce qui s'occupent des commerces sur l'ensemble du territoire de la municipalité. Néanmoins, il y a quelques projets à l'agenda municipal (tableau 4). Par ailleurs, un changement de zonage est proposé dans le plan d'urbanisme en cours de réalisation afin de donner une vocation touristique au centre-ville. Ce plan devrait entrer en vigueur au cours de l'année 2005. La Ville de Roberval attend le nouveau schéma d'aménagement et de développement de la MRC avant de mettre en application les changements prévus. Au surplus, les fêtes du $150^{\mathrm{e}}$ anniversaire de la fondation de la municipalité favoriseront l'animation du centre-ville de Roberval tout au long de 2005. Diverses activités sociales et communautaires s'ajouteront aux traditionnelles festivités du mois de juillet associées à la tenue de la compétition sportive de la traversée à la nage du lac Saint-Jean. 


\section{Tableau 4 - Liste des réalisations récentes et des projets au centre-ville de Roberval}

\begin{tabular}{cl}
\hline Étape & \multicolumn{1}{c}{ Projet } \\
\hline Réalisé & $\begin{array}{l}\text { Enfouissement des réseaux câblés au } \\
\text { centre-ville }\end{array}$ \\
\hline Réalisé & $\begin{array}{l}\text { Construction d’un supermarché MAXI de } \\
40000 \text { pieds carrés }\end{array}$ \\
\hline Réalisé & Aménagement du village des artisans \\
\hline Projet & $\begin{array}{l}\text { Construction d'une nouvelle caserne de } \\
\text { sécurité-incendie }\end{array}$ \\
\hline Projet & $\begin{array}{l}\text { Construction de la Maison de la culture } \\
\text { (ancien couvent des Ursulines) }\end{array}$ \\
\hline Projet & Rénovation de la boulangerie Perron \\
\hline
\end{tabular}

\section{La comparaison des projets de revitalisation}

Comme nous pouvons le constater, les villes du LacSaint-Jean sont fort actives en matière de revitalisation des centres-villes, en particulier Alma, DolbeauMistassini et Saint-Félicien. Plusieurs projets sont sur la table et ils s'inscrivent généralement dans la continuité historique de multiples interventions, plus spécifiquement à Alma et à Dolbeau-Mistassini. Au-delà de leurs spécificités, les plans d'action en revitalisation urbaine d'Alma, de Dolbeau-Mistassini, de SaintFélicien et de Roberval affichent un grand nombre de similitudes.

Premièrement, les élus locaux se montrent sensibles aux besoins des centres-villes, mais leurs politiques peuvent être perçues comme étant contradictoires, car elles misent à la fois sur les développements périurbains et sur la revitalisation du centre. En outre, la compétition entre les villes de Roberval, de SaintFélicien et de Dolbeau-Mistassini en vue d'accueillir un magasin Wal-Mart dans le « haut du Lac » illustre l'attrait persistant des magasins à grande surface. Cependant, il faut admettre que la taille modeste de ces villes fait en sorte que le centre-ville n'est pas toujours précisément délimité et que les développements périphériques sont en réalité assez proches du centre. La dualité centre-périphérie se révèle donc un concept plutôt artificiel dans plusieurs villes petites et moyennes. Mentionnons les cas des espaces commerciaux du boulevard Marcotte à Roberval et de la $8^{\mathrm{e}}$ Avenue à Dolbeau-Mistassini ${ }^{13}$.
Dans un deuxième temps, l'approche de revitalisation utilisée par les administrations locales peut être qualifiée d'opportuniste dans le sens où l'on tente de tirer avantage des programmes et projets des gouvernements supérieurs. En parallèle, on identifie peu de projets privés significatifs au sein des espaces centraux des villes étudiées, les centres-villes étant soutenus principalement par la présence d'hôtels de ville, de palais de justice, d'institutions scolaires ou religieuses et de quelques dizaines de commerces sur rue. Toutefois, la controverse sur le possible déménagement du CLSC d'Alma du centre-ville vers la périphérie a permis de mesurer la volonté de l'administration municipale d'Alma de maintenir les services publics au centre-ville.

\section{II n'y a pas d'énoncés formels reflétant une vision particulière du rôle des centres-villes au sein des villes petites et moyennes.}

En troisième lieu, les programmes de revitalisation jeannois sont essentiellement axés sur des partenariats entre la municipalité et les associations de commerçants. À Alma, on propose même un processus permanent de concertation entre les acteurs commerciaux. ${ }^{14}$ Il s’agit d'un élément positif. Cependant, il n’y a pas de mécanismes ouverts de consultation engageant d'autres intervenants locaux ou la population dans son ensemble au sein des quatre villes à l'étude. En conséquence, il ne faut pas se surprendre que les propositions abordent le centre-ville en termes de vitalité commerciale ou de mise en état des infrastructures ou du mobilier urbain. Très peu de propositions concourent à faire de cet espace un milieu de vie habité et animé. Le centre-ville semble poser problème dans la mesure où des investissements privés sont en danger et que les regroupements de commerçants réclament des actions.

Finalement, il n’y a pas d'énoncés formels reflétant une vision particulière du rôle des centres-villes au sein des villes petites et moyennes, du moins selon nos sources d'information. Au-delà de la rénovation des façades, les villes du Lac-Saint-Jean n'ont pas de programme étoffé favorisant une atmosphère urbaine au centre-ville ou le développement de la fonction résidentielle ${ }^{15}$, à l'exception des résidences pour personnes âgées. Il peut sembler illusoire de s'attaquer 
aux problèmes des centres-villes sans vision globale de la dynamique de ces espaces urbains et sans développer un discours cohérent sur la fonction de ceuxci. La revalorisation culturelle des centres-villes paraît pourtant être un instrument important dans les mécanismes de revitalisation urbaine en contexte nord-américain. Selon nous, il faut soumettre l'enjeu de l'avenir du centre-ville aux débats publics dans le champ politique local.

\section{La revitalisation urbaine intégrée au sein des villes petites et moyennes doit tenir compte des erreurs du passé.}

\section{Conclusion}

Un mouvement de revitalisation des centres-villes est actuellement en cours dans toute la région administrative du Saguenay-Lac-Saint-Jean. En effet, en plus des quatre villes jeannoises analysées, le cœur des trois arrondissements de Saguenay - soit Jonquière, Chicoutimi et La Baie -, fait également l'objet de propositions d'aménagement. Cet intérêt pour la dynamisation des espaces urbains centraux n'est pas nouveau, mais rarement avons-nous assisté à un élan aussi important dans une même période de temps. Est-ce que ces plans d'action auront plus de succès et de durabilité que les programmes d'intervention précédents ? Cela dépend dans une large mesure des approches et stratégies adoptées.

La revitalisation urbaine intégrée au sein des villes petites et moyennes doit tenir compte des erreurs du passé. Il faut absolument éviter l'alternance des cycles de déclin et de relance caractéristique de l'histoire récente des centres-villes québécois. Il importe d'appuyer la revitalisation sur une lecture sensible de la réalité urbaine qui englobe celle-ci dans toute sa complexité. Une vision qui interpelle autant les aménagements physiques que le développement résidentiel et commercial ainsi que l'animation communautaire. Les analyses tendent à démontrer l'importance de mettre de l'avant une diversité de projets à l'intérieur d'une stratégie d'ensemble, tout en évitant les recettes standardisées. Il faut également considérer le contexte géographique particulier des quartiers et des municipalités touchés.

Il s’impose de ne pas oublier la relative rareté des ressources financières publiques au sein des villes petites et moyennes afin d'effectuer des choix collectifs bien ciblés. À cet égard, la coexistence des centres d'achats périphériques et du centre-ville est acceptable aux yeux de la population et de la plupart des intervenants. Il y aurait place pour ces deux types d'espace, car ceux-ci peuvent offrir des services distincts et occuper des rôles différents. Néanmoins, leur existence parallèle au sein des villes petites et moyennes soulève la question de l'allocation des ressources publiques et de la difficulté à investir à la fois au centre et en périphérie. Sur le plan social, la montée des centres commerciaux comme espaces de sociabilité laisse présager une transformation de la vie communautaire au sein des communautés locales.

\section{Notes et références}

1 Martin Simard est géographe et urbaniste. Il est professeurchercheur à l'Université du Québec à Chicoutimi et il dirige le module des sciences humaines de cette même institution.

2 Simon Ouellet est assistant de recherche au sein de l'équipe de l'Atlas électronique du Saguenay-Lac-Saint-Jean et étudiant à la maîtrise en études et interventions régionales de l'UQAC.

3 Les villes petites et moyennes correspondent aux établissements comptant entre 5000 et 70000 habitants. Voir à ce sujet Bruneau, P. (2000), «L’archipel urbain québécois. Un nouveau rapport société-espace », dans P. Bruneau (dir.), Le Québec en changement. Entre l'exclusion et l'espérance. Québec, PUQ, p. 29-60.

4 Voir Fillion, P., H. Hoernig, T. Bunting et G. Sands (2004), "The Successful Few. Healthy Downtowns of Small Metropolitan Regions », Journal of the American Planning Association, vol. 70, n 3, p. 328-343.

5 Voir Carmon, N. (1999), « Three Generations of Urban Renewal Policies: Analysis and Policy Implications », Geoforum, vol. 30, p. 145-158.

6 Voir Divay, G. (2004), « La revitalisation urbaine intégrée. L'art de réussir un puzzle complexe », Urbanité, vol. 3, $\mathrm{n}^{\circ} 3$, p. $15-17$.

7 Phénomène de déplacement progressif des populations moins favorisées qui est également appelé Gentrification. Voir aussi Holcom, H.-B. et R.-A. Beauregard, (1980), Revitalizing Cities, Resource publications in geography, American Association of Geographers, New Jersey.

8 Source : Plan stratégique des interventions au centre-ville d'Alma, Service d'urbanisme et de planification socioéconomique, Ville d’Alma, janvier 1996. 
9 Source : Schéma d'aménagement et de développement de la MRC de Lac-Saint-Jean-Est, 2001.

10 Source : Schéma d’aménagement et de développement de la MRC du Domaine-du-Roy, 1999.

11 Source : Le processus de revitalisation du centre-ville de Saint-Félicien, Comité de revitalisation du centre-ville, Ville de Saint-Félicien, novembre 2004.

12 Source : Schéma d'aménagement et de développement de la MRC du Domaine-du-Roy, 1999.
13 Malgré la proximité des pôles commerciaux anciens et nouveaux, ceux-ci constituent des destinations distinctes; conséquemment, il y a peu de synergies entre les deux zones.

14 Voir Côté, C. (2005), « Vers une concertation permanente », journal Progrès-dimanche, édition du 29 mai, p. A18.

16 Signalons que la Ville d'Alma et la Chambre de commerce et d'industrie du Lac-Saint-Jean-Est ont récemment reçu une étude commandée à la firme Leblond, Bouchard, Daniel Arbour et associés qui pourrait contenir des éléments en ce sens. 


\section{Publicité}

Réseau VRM 